Homology, Homotopy and Applications, vol. 8(1), 2006, pp.145-167

\title{
SPLIT EXTENSIONS, SEMIDIRECT PRODUCT AND HOLOMORPH OF CATEGORICAL GROUPS
}

\author{
STEFANO KASANGIAN, GIUSEPPE METERE AND \\ ENRICO M. VITALE
}

(communicated by George Janelidze)

\begin{abstract}
Working in the context of categorical groups, we show that the semidirect product provides a biequivalence between actions and points. From this biequivalence, we deduce a two-dimensional classification of split extensions of categorical groups, as well as the universal property of the holomorph of a categorical group. We also discuss the link between the holomorph and inner autoequivalences.
\end{abstract}

\section{Introduction}

A categorical group is a monoidal groupoid in which the objects are invertible, up to isomorphism, with respect to the tensor product. Using categorical groups, several well-known notions and results which classically hold for groups can be raised to a categorical level. For example, the correspondence between actions of a group $H$ on a group $G$ and split extensions of $G$ by $H$ has a categorical version established by Garzón and Inassaridze [15]. More precisely, following [7], they define a split extension as a diagram of categorical groups and monoidal functors

$$
\mathbb{G} \stackrel{\mathbf{F}}{\longrightarrow} \mathbb{A} \underset{\mathrm{S}}{\stackrel{\mathrm{G}}{\rightleftarrows}} \mathbb{H}
$$

together with a monoidal natural transformation $\varphi: \mathbf{G F} \Longrightarrow 0$ such that $\mathbf{G}$ is a Grothendieck dense fibration, the factorization of $\mathbf{F}$ through the kernel of $\mathbf{G}$ is an equivalence and $\mathbf{G S}=1_{\mathbb{H}}$. Then, they prove that equivalence classes of split extensions bijectively correspond to equivalence classes of actions of $\mathbb{H}$ on $\mathbb{G}$ (where an action of $\mathbb{H}$ on $\mathbb{G}$ is defined as a monoidal functor $\mathbb{H} \rightarrow \mathcal{E} q(\mathbb{G}), \mathcal{E} q(\mathbb{G})$ being the categorical group of monoidal autoequivalences of $\mathbb{G}$ ).

The quoted paper [15] is one of a series of papers devoted to extensions and derivations in the context of categorical groups, see $[\mathbf{6}, \mathbf{7}, \mathbf{8}, \mathbf{9}, \mathbf{1 0}, \mathbf{1 1}, \mathbf{1 2}],[\mathbf{1 5}, \mathbf{1 6}$, $\mathbf{1 7}, \mathbf{1 8}],[\mathbf{2 3}]$; these papers contain a number of examples showing that categorical groups provide a unifying framework for studying classical homological algebra. Despite its interest, the paper [15] suffers from a mixture of one-dimensional and two-dimensional arguments, and the aim of this note is to put some of the results

Received July 28, 2005, revised September 30, 2005; published on November 28, 2005. 2000 Mathematics Subject Classification: 18D05, 18D10, 18D35, 18G50.

Key words and phrases: Categorical groups, semidirect product, split extensions, holomorph.

Copyright (C) 2005, International Press. Permission to copy for private use granted. 
by Garzón and Inassaridze into their proper 2-categorical context. More precisely, the aim of this note is to improve the results by Garzón and Inassaridze, observing that:

- The assumption that $\mathbf{G}: \mathbb{A} \rightarrow \mathbb{H}$ is a Grothendieck fibration can be avoided and, consequently, the equation $\mathbf{G S}=1_{\mathbb{H}}$ can be replaced by an invertible 2cell. The notion of morphism of extensions can also be weakened, and (split) extensions can be organized in a 2-category (in fact, a 2-groupoid).

- The bijection between classes of split extensions and actions is induced by a biequivalence between the 2-category of split extensions and a locally discrete 2-category of actions. (Let us point out here that in [23, Theorem 2], Rousseau establishes a complete classification of not necessarily split extensions of categorical groups. Rousseau's classification is based on the notion of monoidal fibration of bitorsors and is quite far from what we propose here for split extensions.)

- The biequivalence between split extensions and actions is just the restriction of a more fundamental biequivalence between the 2-category of actions and a suitable 2-category whose objects are "points", i.e. diagrams of categorical groups of the form

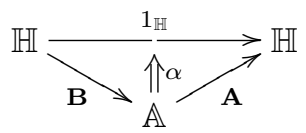

Moreover, we introduce the holomorph of a categorical group $\mathbb{G}$ as the semidirect product

$$
\mathcal{H o l} \mathbb{G}=\mathbb{G} \rtimes \mathcal{E} q(\mathbb{G})
$$

(where the action $\mathcal{E} q(\mathbb{G}) \times \mathbb{G} \rightarrow \mathbb{G}$ is the evaluation) and we deduce the universal property of $\mathcal{H o l} \mathbb{G}$ directly from the biequivalence between split extensions and actions. Finally, we explain in what sense the holomorph is the universal solution to the problem of converting autoequivalences into inner autoequivalences.

The paper is organized as follows. In Section 2, for the convenience of the reader, we recall the classification of split extensions of groups following the modern approach due to Bourn and Janelidze [5], [19]. We also discuss various universal properties of the holomorph; the first of them has been studied in the more general context of semi-abelian categories by Borceux, Janelidze and Kelly in [3], [4]. In Section 3, we recall basic definitions and properties of categorical groups and fix notations. The content of Section 4 is the biequivalence between points and actions, and its restriction to split extensions. The final section is devoted to the holomorph of a categorical group. 


\section{The case of groups}

\subsection{The equivalence between actions and points}

Let us first describe the category Act of actions in the category Grp of groups. An object is given by a group $G$, a group $H$ and an action of $H$ on $G$, that is, a map

$$
H \times G \rightarrow G \quad(x, a) \mapsto{ }^{x} a
$$

such that ${ }^{x \cdot y} a={ }^{x}\left({ }^{y} a\right),{ }^{1} a=a$ and ${ }^{x}(a+b)={ }^{x} a+{ }^{x} b$ (we use multiplicative notation for $H$ and additive notation for $G$ ). Given two actions $H \times G \rightarrow G$ and $H^{\prime} \times$ $G^{\prime} \rightarrow G^{\prime}$, a morphism is a pair of equivariant group homomorphisms $g: G \rightarrow G^{\prime}$ and $h: H \rightarrow H^{\prime}$, that is, $g\left({ }^{x} a\right)={ }^{h(x)} g(a)$.

We pass now to the category $\mathbf{P t}$ of points in the category of groups. An object is a diagram of groups and homomorphisms

$$
A \underset{\beta}{\stackrel{\alpha}{\rightleftarrows}} H
$$

such that $\alpha \beta=1_{H}$. An arrow from $(A, H, \alpha, \beta)$ to $\left(A^{\prime}, H^{\prime}, \alpha^{\prime}, \beta^{\prime}\right)$ is a pair of group homomorphisms $f: A \rightarrow A^{\prime}$ and $h: H \rightarrow H^{\prime}$ such that $f \beta=\beta^{\prime} h$ and $h \alpha=\alpha^{\prime} f$.

There is a functor

$$
\mathcal{K}: \mathbf{P t} \rightarrow \text { Act }
$$

which sends a point $(A, H, \alpha, \beta)$ to the action of $H$ on $\mathcal{K} e r \alpha$, the kernel of $\alpha$, given by

$$
H \times \mathcal{K} \operatorname{er} \alpha \rightarrow \mathcal{K} \operatorname{er} \alpha \quad(x, a) \mapsto \beta(x)+a-\beta(x) .
$$

This functor is an equivalence, with quasi-inverse given by semidirect product

$$
\rtimes: \text { Act } \rightarrow \text { Pt. }
$$

Indeed, if $H \times G \rightarrow G$ is an action, the semidirect product $G \rtimes H$ is the cartesian product $G \times H$ equipped with a group structure given by $(a, x)+(b, y)=$ $\left(a+{ }^{x} b, x \cdot y\right), 0=(0,1),-(a, x)=\left(-x^{-1} a, x^{-1}\right)$. Moreover, we get a point

$$
G \rtimes H \underset{i}{\stackrel{p}{\gtrless}} H
$$

with $p(a, x)=x$ and $i(x)=(0, x)$.

\subsection{The classification of split extensions}

There are two forgetful functors:

$$
\begin{array}{rl}
\mathcal{U}_{*}: \mathbf{P t} \rightarrow \mathbf{G r p} & (A, H, \alpha, \beta) \mapsto H \\
\mathcal{O}: \mathbf{A c t} \rightarrow \mathbf{G r p} & H \times G \rightarrow G \mapsto H
\end{array}
$$

making the diagram

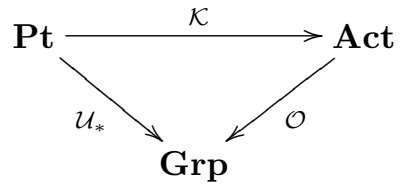


commutative. If we fix a group $H$ and consider the fibers over $H$ of these forgetful functors, the equivalence $\mathcal{K}$ restricts to an equivalence $\mathcal{K}^{\prime}: \mathbf{P t}(H) \rightarrow \operatorname{Act}(H)$.

Now we can define two other functors:

$$
\begin{array}{rlrl}
\mathcal{N}: \operatorname{Pt}(H) & \rightarrow \operatorname{Grp} & & (A, \alpha, \beta) \mapsto \mathcal{K} e r \alpha \\
\mathcal{U}: \operatorname{Act}(H) & \rightarrow \operatorname{Grp} & H \times G \rightarrow G \mapsto G
\end{array}
$$

Once again, the diagram

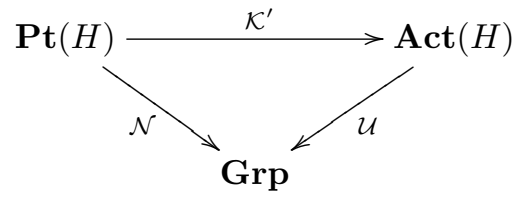

commutes and, if we fix a group $G$, the equivalence $\mathcal{K}^{\prime}$ restricts to an equivalence

$$
\mathcal{K}^{\prime \prime}: \mathbf{E x t}_{\text {split }}(H, G) \rightarrow \operatorname{Act}(H, G)
$$

on the fibers. Explicitly, $\operatorname{Act}(H, G)$ is the set (=discrete category) of actions of $H$ on $G$, and $\mathbf{E x t}_{\text {split }}(H, G)$ is the groupoid whose objects are split extensions of $G$ by $H$, that is, points $(A, H, \alpha, \beta)$ such that $G$ is the kernel of $\alpha$, and arrows are homomorphisms $f: A \rightarrow A^{\prime}$ such that $\alpha^{\prime} f=\alpha, f \beta=\beta^{\prime}$ and inducing the identity on $G$.

\subsection{First universal property of the holomorph}

If we denote by $\operatorname{EXT}_{\text {split }}(H, G)$ the set of isomorphism classes of split extensions, the equivalence $\mathcal{K}^{\prime \prime}: \mathbf{E x t}_{\text {split }}(H, G) \rightarrow \operatorname{Act}(H, G)$ induces a bijection

$$
\operatorname{EXT}_{\text {split }}(H, G) \simeq \operatorname{Act}(H, G) \text {. }
$$

Moreover, $\operatorname{Act}(H, G)$ is also in bijection with the set of group homomorphisms from $H$ to the group of automorphisms of $G$

$$
\operatorname{Act}(H, G) \simeq \operatorname{Hom}(H, \mathcal{A} u t G) .
$$

Putting together these bijections and letting the group $H$ vary, we get a natural isomorphism of functors:

$$
\theta: \operatorname{EXT}_{\text {split }}(-, G) \Longrightarrow \operatorname{Hom}(-, \mathcal{A} u t G): \mathbf{G r p}^{o p} \rightarrow \mathbf{S e t},
$$

where $\operatorname{EXT}_{\text {split }}(-, G)$ is defined on a morphism $h: H \rightarrow H^{\prime}$ by the following pullback

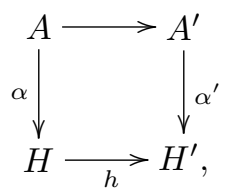

which is a split-pullback; the section $\beta$ of $\alpha$ is the one induced by the section $\beta^{\prime}$ of $\alpha^{\prime}$.

It is a general fact, coming from Yoneda's lemma, that a functor $F: \mathcal{C}^{o p} \rightarrow$ Set is representable, that is, there is an object $R \in \mathcal{C}$ and a natural isomorphism 
$\theta: F \Longrightarrow \mathcal{C}(-, R)$, if and only if there is an element $a \in F(R)$, given by $\theta_{R}^{-1}\left(1_{R}\right)$, satisfying the following universal property: for all $H \in \mathcal{C}$ and for all $x \in F(H)$, there is a unique $h: H \rightarrow R$ such that $x=F(h)(a)$.

If we apply this general fact to $\theta: \operatorname{EXT}_{\text {split }}(-, G) \Longrightarrow \operatorname{Hom}(-, \mathcal{A} u t G)$, we get as element $\theta^{-1}\left(1_{\mathcal{A u t} G}\right)$ the (isomorphism class of $)$ the holomorph $\mathcal{H o l}(G)=G \rtimes \mathcal{A} u t G$ of $G$

$$
G \stackrel{i n_{1}}{\longrightarrow} G \rtimes \mathcal{A} u t G \underset{i n_{2}}{\stackrel{p r}{\rightleftarrows}} \mathcal{A} u t G
$$

Accordingly, the following is its universal property: for any group $H$ and for any split extension $G \longrightarrow A \rightleftarrows H$, there is a unique homomorphism $h: H \rightarrow \mathcal{A} u t G$ such that $A \rightleftarrows H$ is the split-pullback of $G \rtimes \mathcal{A} u t G \longleftrightarrow \mathcal{A} u t G$ along $h$.

\subsection{Second universal property of the holomorph}

Since inner automorphisms are easier to handle than general automorphisms, a natural question is if a group $G$ can be embedded into a group $G^{\prime}$ in such a way that each automorphism of $G$ is the restriction of an inner automorphism of $G^{\prime}$. This is the way the holomorph is classically introduced [22]: the canonical injections

$$
G \stackrel{i n_{1}}{\longrightarrow} \mathcal{H o l}(G)=G \rtimes \mathcal{A u t}(G){\stackrel{i n_{2}}{\longleftarrow}}^{A u t}(G)
$$

are such that the diagram

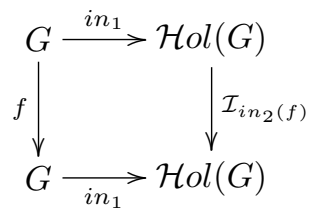

commutes for any $f \in \mathcal{A u t}(G)$, where $\mathcal{I}_{\text {in }_{2}(f)} \in \mathcal{A} u t(\mathcal{H o l}(G))$ is the inner automorphism associated with the element $i n_{2}(f) \in \mathcal{H o l}(G)$. In fact, more is true: the holomorph is the universal solution for such a problem. Given a group $A$ and two morphisms

$$
G \stackrel{g}{\longrightarrow} A \stackrel{h}{\longleftarrow} \mathcal{A u t}(G)
$$

such that, for every $f \in \mathcal{A} u t(G)$, the diagram

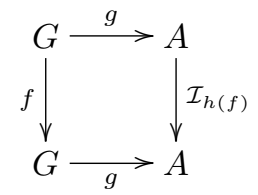

commutes, there is a unique morphism $\gamma: \mathcal{H o l}(G) \rightarrow A$ such that $\gamma \cdot i n_{1}=g$ and $\gamma \cdot i n_{2}=h$ (just put $\gamma(x, f)=g(x) h(f)$ for $x \in G, f \in \mathcal{A} u t(G)$ ). 
This second universal property of the holomorph is a special case of a more general fact: the functor

$$
\rtimes: \text { Act } \rightarrow \text { Grp }
$$

has a right adjoint

$$
\mathcal{C}: \operatorname{Grp} \rightarrow \text { Act }
$$

which associates to a group $A$ the conjugation $A \times A \rightarrow A:(x, y) \mapsto x y x^{-1}$. Indeed, equivariant pairs $(g: G \rightarrow A, h: H \rightarrow A$ ) bijectively correspond to group homomorphisms $\gamma: G \rtimes H \rightarrow A$ : given $(g, h)$, put $\gamma(a, x)=g(a) h(x)$; conversely, precompose $\gamma$ with the canonical injections in the semidirect product. Now, if one specializes this situation taking $\mathcal{A} u t(G)$ as group $H$ and the evaluation $\mathcal{A} u t(G) \times G \rightarrow G$ as action, then the condition on $(g, h)$ to be equivariant precisely says that for all $f \in \mathcal{A} u t(G)$, diagram (1) commutes. (Note that, via the equivalence $\mathbf{A c t} \simeq \mathbf{P t}$, the adjunction $\rtimes \dashv \mathcal{C}$ corresponds to the adjunction $\mathcal{U}^{*} \dashv \Delta: \mathbf{P t} \rightarrow \operatorname{Grp}$, where $\mathcal{U}^{*}(A, H, \alpha, \beta)=A$ and $\Delta(A)$ is the diagonal point.)

\subsection{Holomorph and the Mal'tsev operation}

The holomorph of a group $G$ can be described also via the canonical Mal'tsev operation. For the sake of completeness, we recall this description here, but leave the generalization to categorical groups as an exercise for the reader. Recall that the canonical Mal'tsev operation on $G$ is the ternary operation defined by

$$
m: G \times G \times G \rightarrow G \quad m(x, y, z)=x-y+z
$$

Let us call $\operatorname{Mal}(G)$ the subgroup of the group of transformations on $G$ given by those bijections $t: G \rightarrow G$ such that $t(x-y+z)=t(x)-t(y)+t(z)$. There is an equivariant pair

$$
g: G \rightarrow \operatorname{Mal}(G) \quad g_{x}(y)=x+y ; \quad h: \operatorname{Aut}(G) \rightarrow \operatorname{Mal}(G) \quad h=\text { inclusion. }
$$

By the second universal property of the holomorph, from the pair $(g, h)$, we get a morphism

$$
\gamma: \mathcal{H o l}(G) \rightarrow \operatorname{Mal}(G) \quad \gamma(x, f)=g_{x} \cdot f .
$$

The morphism $\gamma$ is an isomorphism: if we put $t=g_{x} \cdot f$, we can obtain $x$ and $f$ using the formulae $x=t(0)$ and $f=g_{t(0)}^{-1} \cdot t$.

\section{Preliminaries on categorical groups}

\subsection{The 2-category of categorical groups}

Basic facts on categorical groups can be found in $[\mathbf{1}, \mathbf{7}, \mathbf{1 4}, \mathbf{2 0}, \mathbf{2 1}, \mathbf{2 4}, \mathbf{2 6}]$. Let us recall that a categorical group $\mathbb{G}=(\mathbb{G}, \otimes, I, \ldots)$ is a monoidal groupoid in which the objects are invertible, up to isomorphism, with respect to the tensor product. This means that for each object $A$ in $\mathbb{G}$, there is an object $A^{*}$ and a morphism $\epsilon_{A}: A^{*} \otimes A \rightarrow I$. One can then choose a morphism $\eta_{A}: I \rightarrow A \otimes A^{*}$ so that $\left(A^{*}, A, \eta_{A}, \epsilon_{A}\right)$ is a duality. Extending this to arrows, one gets an op-monoidal equivalence $\mathbb{G} \rightarrow \mathbb{G}^{o p}$. Categorical groups are the objects of a 2 -category $\mathbf{C G}$. Homomorphisms of categorical groups are just monoidal functors, and 2-cells are monoidal 
natural transformations (which always are natural isomorphisms). A point of notation: if $\mathbf{F}: \mathbb{G} \rightarrow \mathbb{H}$ is a monoidal functor, its monoidal structure is denoted by $\mu^{\mathbf{F}}=\mu_{X, Y}^{\mathbf{F}}: F(X \otimes Y) \rightarrow F(X) \otimes F(Y)$ (a canonical arrow $\mu_{0}=\mu_{0}^{\mathbf{F}}: F(I) \rightarrow I$ can be deduced).

\subsection{The 2-category of split extensions}

The kernel $\left(\mathcal{N}(\mathbf{F}), \mathbf{e}_{\mathbf{F}}, \lambda^{\mathbf{F}}\right)$ of a homomorphism $\mathbf{F}: \mathbb{G} \rightarrow \mathbb{H}$ (see [21]) is the universal solution, in the sense of a bilimit, to the problem of finding a categorical group $\mathbb{K}$, a homomorphism $\mathbf{G}$ and a 2-cell $\phi$ as in the following diagram, where 0 is the functor sending each arrow of $\mathbb{K}$ on the identity of the unit object of $\mathbb{H}$,

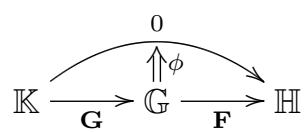

An explicit description of the kernel is provided by the comma-category whose objects are pairs $(A \in \mathbb{G}, a: F(A) \rightarrow I)$ and whose arrows are arrows $f: A \rightarrow B$ in $\mathbb{G}$ such that $a=b F(f)$. The monoidal structure of $\mathcal{N}(\mathbf{F})$ is defined from those of $\mathbb{G}$ and $\mathbf{F}$ in the only sensible way. The functor $\mathbf{e}_{\mathbf{F}}: \mathcal{N}(\mathbf{F}) \rightarrow \mathbb{G}$ is the canonical forgetful functor, and the 2-cell $\lambda^{\mathbf{F}}: \mathbf{F e}_{\mathbf{F}} \Rightarrow 0$ is $\lambda_{(A, a)}^{\mathbf{F}}=a$.

Using kernels, we can define 2-exactness and extensions [21], [23].

Definition 3.1. Consider the following diagram in $\mathbf{C G}$

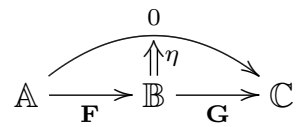

1. The triple $(\mathbf{F}, \eta, \mathbf{G})$ is 2-exact if the factorization of $\mathbf{F}$ through the kernel of $\mathbf{G}$ is a full and essentially surjective functor.

2. The 2-exact triple $(\mathbf{F}, \eta, \mathbf{G})$ is an extension of categorical groups if $\mathbf{F}$ is faithful and $\mathbf{G}$ is essentially surjective on objects.

Observe that if $(\mathbf{F}, \eta, \mathbf{G})$ is an extension, the factorization of $\mathbf{F}$ through the kernel of $\mathbf{G}$ is an equivalence.

Extensions of a categorical group $\mathbb{C}$ by a categorical group $\mathbb{A}$ are the objects of the 2-category $\operatorname{Ext}(\mathbb{C}, \mathbb{A})$. If $(\mathbf{F}, \eta, \mathbf{G})$ and $\left(\mathbf{F}^{\prime}, \eta^{\prime}, \mathbf{G}^{\prime}\right)$ are two such extensions, an arrow between them is a triple $(\sigma, \mathbf{E}, \tau)$ in $\mathbf{C G}$ as in the following diagram,

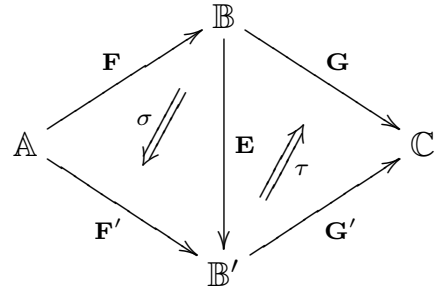

with $\sigma$ and $\tau$ compatible with $\eta$ and $\eta^{\prime}$. Finally, if $(\sigma, \mathbf{E}, \tau)$ and $\left(\sigma^{\prime}, \mathbf{E}^{\prime}, \tau^{\prime}\right)$ are arrows as above, a 2-cell $\theta$ between them is a 2-cell $\theta: \mathbf{E} \Longrightarrow \mathbf{E}^{\prime}$ in $\mathbf{C G}$ compatible with $\sigma, \sigma^{\prime}$ 
and with $\tau, \tau^{\prime}$. In analogy with the case of groups, the 2-category $\operatorname{Ext}(\mathbb{C}, \mathbb{A})$ is a 2-groupoid [23], [6]: 1-cells are equivalences and 2-cells are isomorphisms.

For our purposes, we are interested in a sub-2-category of $\operatorname{Ext}(\mathbb{C}, \mathbb{A})$, namely, that of split extensions. A split extension is an extension for which the epi part admits a (not necessarily strict) section in $\mathbf{C G}$. More precisely, a split extension is a 5 -tuple $(\mathbf{F}, \eta, \mathbf{G}, \mathbf{S}, \varsigma)$, where $(\mathbf{F}, \eta, \mathbf{G})$ is a categorical group extension, $\mathbf{S}: \mathbb{C} \rightarrow \mathbb{B}$ is a homomorphism and $\varsigma: \mathbf{G S} \Longrightarrow 1_{\mathbb{C}}$ is a 2-cell in $\mathbf{C G}$. An arrow between two split extensions is a 4 -tuple $(\sigma, \mathbf{E}, \tau, s):(\mathbf{F}, \eta, \mathbf{G}, \mathbf{S}, \varsigma) \rightarrow\left(\mathbf{F}^{\prime}, \eta^{\prime}, \mathbf{G}^{\prime}, \mathbf{S}^{\prime}, \varsigma^{\prime}\right)$, where $(\sigma, \mathbf{E}, \tau)$ is an arrow in $\operatorname{Ext}(\mathbb{C}, \mathbb{A})$, and $s: \mathbf{E S} \Longrightarrow \mathbf{S}^{\prime}$ is a 2-cell of $\mathbf{C G}$ making commutative the following diagram

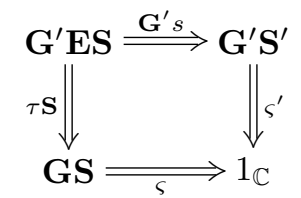

A 2-cell $\theta:(\sigma, \mathbf{E}, \tau, s) \rightarrow\left(\sigma^{\prime}, \mathbf{E}^{\prime}, \tau^{\prime}, s^{\prime}\right)$ is a 2-cell of $\mathbf{E x t}(\mathbb{C}, \mathbb{A})$ compatible with $s, s^{\prime}$. The 2-category of split categorical group extensions of $\mathbb{C}$ by $\mathbb{A}$ will be denoted by $\operatorname{Ext}_{\text {split }}(\mathbb{C}, \mathbb{A})$.

\subsection{Actions and semidirect product}

Let $\mathbb{H}$ be a categorical group. Recall from [15] that an $\mathbb{H}$-categorical group is a categorical group $\mathbb{G}$ equipped with a homomorphism

$$
\Sigma: \mathbb{H} \rightarrow \mathcal{E} q(\mathbb{G})
$$

or, equivalently, equipped with an action, that is, a functor

$$
a c: \mathbb{H} \times \mathbb{G} \rightarrow \mathbb{G}, \quad(X, A) \mapsto{ }^{X} A=\Sigma_{X}(A)
$$

and three natural isomorphisms

$$
\begin{gathered}
\psi=\psi_{X, A, B}:{ }^{X}(A \otimes B) \rightarrow{ }^{X} A \otimes{ }^{X} B, \quad \phi=\phi_{X, Y, A}:{ }^{X \otimes Y} A \rightarrow{ }^{X}\left({ }^{Y} A\right), \\
\phi_{0}=\phi_{0, A}:{ }^{I} A \rightarrow A
\end{gathered}
$$

satisfying suitable coherence conditions. Note that a categorical group always acts on itself by conjugation [7], [23]

$$
\mathcal{I}: \mathbb{G} \rightarrow \mathcal{E} q(\mathbb{G})
$$

where $\mathcal{I}_{X}$ is the monoidal auto-equivalence $A \mapsto(X \otimes A) \otimes X^{*}$.

Let $\mathbb{G}$ be an $\mathbb{H}$-categorical group. The semidirect product $\mathbb{G} \rtimes \mathbb{H}$ has the product $\mathbb{G} \times \mathbb{H}$ as underlying groupoid. The tensor product is $(A, X) \otimes(B, Y)=(A \otimes$ $\left.{ }^{X} B, X \otimes Y\right)$, and constraints and inverses are constructed using $\psi, \phi, \phi_{0}$ and those of $\mathbb{G}$ and $\mathbb{H}$. Semidirect product provides the typical example of split extension. Indeed,

$$
(\mathbf{Q},=, \mathbf{P}, \mathbf{S},=): \mathbb{G} \stackrel{\mathbf{Q}}{\longrightarrow} \mathbb{G} \rtimes \mathbb{H} \stackrel{\mathbf{P}}{\underset{\mathbf{S}}{\longrightarrow}} \mathbb{H}
$$

is a split extension, where $\mathbf{Q}, \mathbf{S}, \mathbf{P}$ are the canonical injections and projections. 


\section{Actions and points}

In this section, we introduce the 2-category $\mathbf{P t}(\mathbf{C G})$ of points in $\mathbf{C G}$ and the 2-category $\mathbf{A c t}(\mathbf{C G})$ of categorical group actions and prove that they are biequivalent.

\subsection{The general setting}

The 2-category $\mathbf{P t}(\mathbf{C G})$ consists of the following data: objects are 5-tuples $(\mathbb{A}, \mathbb{H}, \mathbf{A}, \mathbf{B}, \alpha)$ in $\mathbf{C G}$ :

$$
\mathbb{A} \stackrel{\mathbf{A}}{\underset{\mathbf{B}}{\rightleftarrows}} \mathbb{H}, \quad \alpha: \mathbf{A B} \Longrightarrow \mathbf{1}_{\mathbb{H}} .
$$

Arrows are 4-tuples $(\mathbf{F}, \mathbf{H}, \varphi, \chi):(\mathbb{A}, \mathbb{H}, \mathbf{A}, \mathbf{B}, \alpha) \rightarrow\left(\mathbb{A}^{\prime}, \mathbb{H}^{\prime}, \mathbf{A}^{\prime}, \mathbf{B}^{\prime}, \alpha^{\prime}\right)$ in $\mathbf{C G}$ :

$$
F: \mathbb{A} \rightarrow \mathbb{A}^{\prime}, \quad H: \mathbb{H} \rightarrow \mathbb{H}^{\prime}, \quad \varphi: \mathbf{F B} \Longrightarrow \mathbf{B}^{\prime} \mathbf{H}, \quad \chi: \mathbf{A}^{\prime} \mathbf{F} \Longrightarrow \mathbf{H A}
$$

such that the following diagram commutes

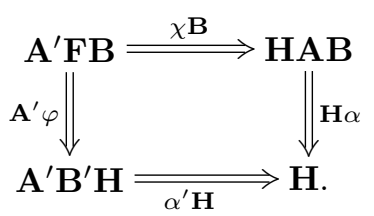

Finally, a 2-cell $(\delta, \epsilon):(\mathbf{F}, \mathbf{H}, \varphi, \chi) \Longrightarrow\left(\mathbf{F}^{\prime}, \mathbf{H}^{\prime}, \varphi^{\prime}, \chi^{\prime}\right)$ is a pair of 2-cells $\delta: \mathbf{F} \Longrightarrow \mathbf{F}^{\prime}$ and $\epsilon: \mathbf{H} \Longrightarrow \mathbf{H}^{\prime}$ in $\mathbf{C G}$ such that the following diagrams commute
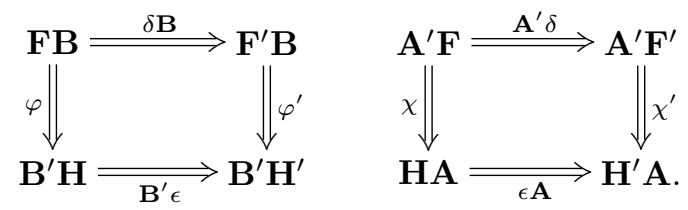

Extending the definition of the 2-category of $\mathbb{H}$-categorical groups, given in [11], in order to let $\mathbb{H}$ change, we get the 2-category $\mathbf{A c t}(\mathbf{C G})$ of categorical groups actions. It consists of the following data: the objects are categorical group actions; a morphism between the $\mathbb{H}$-categorical group $\mathbb{G}$ and the $\mathbb{H}^{\prime}$-categorical group $\mathbb{G}^{\prime}$ is a pair of homomorphisms

$$
\mathbf{R}: \mathbb{H} \rightarrow \mathbb{H}^{\prime} \quad \mathbf{T}: \mathbb{G} \rightarrow \mathbb{G}^{\prime}
$$

in CG, equivariant with respect to the actions, that is, equipped with a natural transformation $\rho$

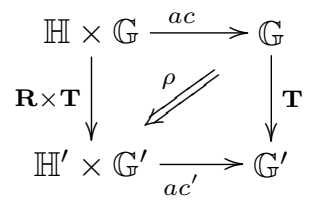


making the following diagrams commute:
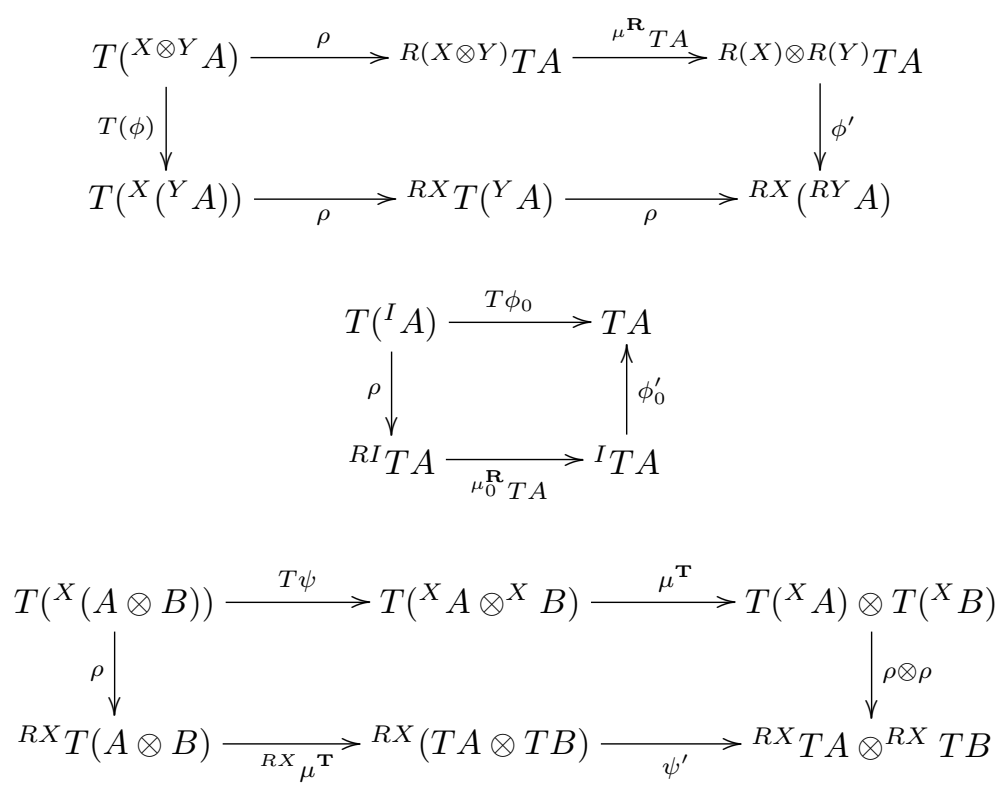

Finally, a 2-cell $(\mathbf{R}, \mathbf{T}, \rho) \Longrightarrow\left(\mathbf{R}^{\prime}, \mathbf{T}^{\prime}, \rho^{\prime}\right)$ is a pair of 2-cells $\alpha: \mathbf{R} \Longrightarrow \mathbf{R}^{\prime}$ and $\beta: \mathbf{T} \Longrightarrow \mathbf{T}^{\prime}$ in $\mathbf{C G}$ such that the following diagram commutes:

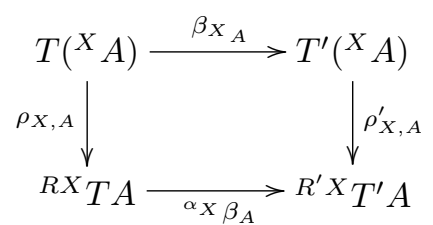

\subsection{The main biequivalence}

The semi-direct product described in Section 3.3 is part of a 2 -functor

$$
\rtimes: \operatorname{Act}(\mathbf{C G}) \rightarrow \mathbf{P t}(\mathbf{C G}),
$$

which may be viewed as follows:

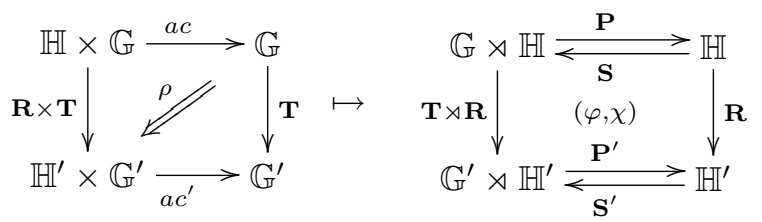

More precisely, given an action $a c: \mathbb{H} \times \mathbb{G} \rightarrow \mathbb{G}$, its image is the point

$$
\mathbb{G} \rtimes \mathbb{H} \underset{\mathbf{S}}{\stackrel{\mathbf{P}}{\rightleftarrows}} \mathbb{H}, \quad \text { PS }=1_{\mathbb{H}}
$$


Given a 1-cell $(\mathbf{R}, \mathbf{T}, \rho)$ from $\mathbb{H} \times \mathbb{G} \rightarrow \mathbb{G}$ to $\mathbb{H}^{\prime} \times \mathbb{G}^{\prime} \rightarrow \mathbb{G}^{\prime}$ in $\operatorname{Act}(\mathbf{C G})$, we get a 1-cell $(\mathbf{T} \rtimes \mathbf{R}, \mathbf{R}, \varphi, \chi)$ in $\mathbf{P t}(\mathbf{C G})$, with

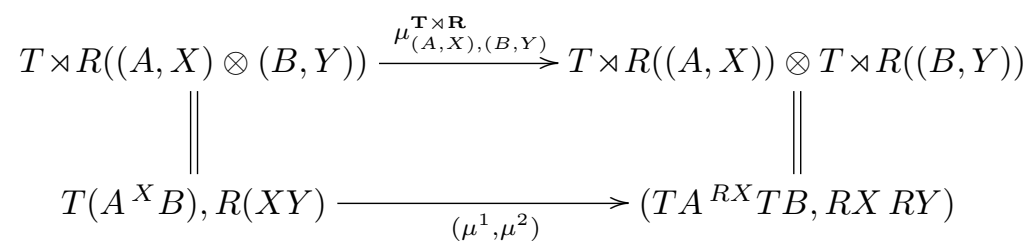

(where $\mu^{1}=\left(T A \otimes\left(\rho_{X, B}\right)\right) \circ \mu_{A, X_{B}}^{\mathbf{T}}$ and $\left.\mu^{2}=\mu_{X, Y}^{\mathbf{R}}\right) \varphi_{X}=\left(\mu_{0}^{\mathbf{T}}, i d\right):(T I, R X) \rightarrow$ $(I, R X)$ and $\chi_{(A, X)}=i d: R X \rightarrow R X$. Finally, the image of a 2-cell $(\alpha, \beta)$ of $\operatorname{Act}(\mathbf{C G})$ is the 2-cell $((\beta, \alpha), \alpha)$ of $\mathbf{P t}(\mathbf{C G})$.

Proposition 4.1. The 2-functor

$$
\rtimes: \operatorname{Act}(\mathbf{C G}) \rightarrow \operatorname{Pt}(\mathbf{C G})
$$

is a biequivalence of 2-categories.

Proof. We split the proof into two lemmas. Recall that a 2-functor $\mathcal{F}: \mathcal{A} \rightarrow \mathcal{B}$ is a biequivalence if it induces equivalences on hom-categories, and if it is biessentially surjective, that is, for every object $Y$ of $\mathcal{B}$, there exists an object $X$ of $\mathcal{A}$ and an equivalence $\mathcal{F}(X) \rightarrow Y$.

Lemma 4.2. Consider two objects ac: $\mathbb{H} \times \mathbb{G} \rightarrow \mathbb{G}$ and $a c^{\prime}: \mathbb{H}^{\prime} \times \mathbb{G}^{\prime} \rightarrow \mathbb{G}^{\prime}$ in $\operatorname{Act}(\mathbf{C G})$. The induced functor on hom-categories

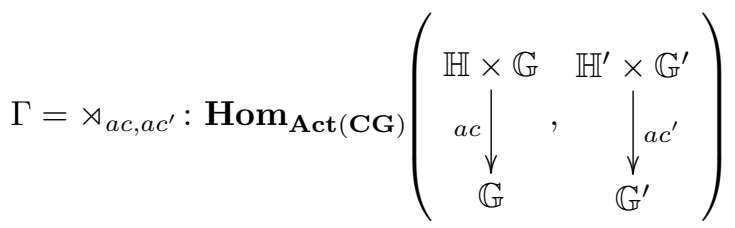

$$
\begin{aligned}
& \rightarrow \operatorname{Hom}_{\mathbf{P t}(\mathbf{C G})}\left(\begin{array}{ccc}
\mathbb{G} \rtimes \mathbb{H} & \mathbb{G}^{\prime} \rtimes \mathbb{H}^{\prime} \\
\mathbf{S} & \left.\uparrow\right|_{\downarrow} \mathbf{P} & \left.\mathbf{S}^{\prime}\right|_{\mathbb{V}} \mathbf{P}^{\prime} \\
\mathbb{H} & \mathbb{H}^{\prime}
\end{array}\right)
\end{aligned}
$$

is an equivalence.

Proof. $\Gamma$ is clearly faithful. As far as fullness is concerned, consider $(\mathbf{R}, \mathbf{T}, \rho)$ and $\left(\mathbf{R}^{\prime}, \mathbf{T}^{\prime}, \rho^{\prime}\right)$ in $\mathbf{H o m}_{\mathbf{A c t}(\mathbf{C G})}\left(a c, a c^{\prime}\right)$, and $(\delta, \epsilon): \Gamma(\mathbf{R}, \mathbf{T}, \rho) \Longrightarrow \Gamma\left(\mathbf{R}^{\prime}, \mathbf{T}^{\prime}, \rho^{\prime}\right)$. Observe that $\delta$ has two components, say

$$
\delta_{A, X}=\left(\delta_{A, X}^{(1)}, \delta_{A, X}^{(2)}\right):(T A, R X) \rightarrow\left(T^{\prime} A, R^{\prime} X\right)
$$

Therefore, we get $\alpha: \mathbf{R} \Longrightarrow \mathbf{R}^{\prime}$ by $\alpha_{X}=\delta_{I, X}^{(2)}$, and $\beta: \mathbf{T} \Longrightarrow \mathbf{T}^{\prime}$ by $\beta_{A}=\delta_{A, I}^{(1)}$. Using that $\delta$ is monoidal, one checks that $(\alpha, \beta):(\mathbf{R}, \mathbf{T}, \rho) \Longrightarrow\left(\mathbf{R}^{\prime}, \mathbf{T}^{\prime}, \rho^{\prime}\right)$ is a 2-cell in $\operatorname{Act}(\mathbf{C G})$. Moreover, using that $(\delta, \epsilon)$ is a 2 -cell in $\mathbf{P t}(\mathbf{C G})$, one checks that $\delta_{A, X}^{(2)}=$ $\epsilon_{X}$ and $\delta_{A, X}^{(1)}=\delta_{(A, I)}^{(1)}$, so that $\Gamma(\alpha, \beta)=(\delta, \epsilon)$. 
We now prove that $\Gamma$ is essentially surjective. Consider

$$
(F, H, \varphi, \chi) \in \mathbf{H o m}_{\mathbf{P t}(\mathbf{C G})}\left(\begin{array}{ccc}
\mathbb{G} \rtimes \mathbb{H} & \mathbb{G}^{\prime} \rtimes \mathbb{H}^{\prime} \\
\mathbf{S} & \uparrow_{\downarrow} \mathbf{P}, & \left.\mathbf{S}^{\prime}\right|_{\downarrow^{\prime}} \\
\mathbb{H} & \mathbb{H}^{\prime}
\end{array}\right)
$$

We look for $(\mathbf{R}, \mathbf{T}, \rho) \in \mathbf{H o m}_{\mathbf{A c t}(\mathbf{C G})}\left(a c, a c^{\prime}\right)$ and a 2-cell $(\delta, \epsilon): \Gamma(\mathbf{R}, \mathbf{T}, \rho) \Longrightarrow$ $(\mathbf{F}, \mathbf{H}, \varphi, \chi)$ in $\mathbf{P t}(\mathbf{C G})$. One can choose $\mathbf{R}=\mathbf{H}: \mathbb{H} \rightarrow \mathbb{H}^{\prime}$ and $\mathbf{T}=F_{1} Q: \mathbb{G} \rightarrow \mathbb{G} \rtimes$ $\mathbb{H} \rightarrow \mathbb{G}^{\prime}$, where $F_{1}, F_{2}$ are the components of $\mathbf{F}: \mathbb{G} \rtimes \mathbb{H} \rightarrow \mathbb{G}^{\prime} \rtimes \mathbb{H}^{\prime}$. The monoidal structure of $\mathbf{T}$ is given by the first component of the following chain of natural isomorphisms:

$$
\begin{gathered}
\left(F_{1}(A, I) \otimes F_{1}(B, I), I\right) \simeq\left(F_{1}(A, I), I\right) \otimes\left(F_{1}(B, I), I\right) \simeq \\
\simeq\left(F_{1}(A, I), F_{2}(A, I)\right) \otimes\left(F_{1}(B, I), F_{2}(B, I)\right)=F(A, I) \otimes F(B, I) \simeq \\
\simeq F((A, I) \otimes(B, I)) \simeq F(A \otimes B, I)=\left(F_{1}(A \otimes B, I), F_{2}(A \otimes B, I)\right),
\end{gathered}
$$

where the second isomorphism is provided by $\chi$, and the other isomorphisms are canonical. Similarly, the 2-cell $\rho$ is the first component of the following chain of natural isomorphisms:

$$
\begin{gathered}
\left(F_{1}\left({ }^{X} A, I\right), F_{2}\left({ }^{X} A, I\right)\right)=F\left({ }^{X} A, I\right) \simeq F\left((I, X) \otimes(A, I) \otimes\left(I, X^{*}\right)\right) \simeq \\
\simeq F(I, X) \otimes F(A, I) \otimes F\left(I, X^{*}\right)= \\
=\left(F_{1}(I, X), F_{2}(I, X)\right) \otimes\left(F_{1}(A, I), F_{2}(A, I)\right) \otimes\left(F_{1}\left(I, X^{*}\right), F_{2}\left(I, X^{*}\right)\right) \simeq \\
\simeq(I, H(X)) \otimes\left(F_{1}(A, I), I\right) \otimes\left(I, H\left(X^{*}\right)\right) \simeq\left({ }^{H(X)} F_{1}(A, I), I\right),
\end{gathered}
$$

where the third isomorphism is provided by $\varphi$ on the first component of the first and third objects, by $\chi$ on the second component of the first and third objects and by $\chi$ again on the second object. The other isomorphisms are canonical. It remains to construct the 2-cell $(\delta, \epsilon)$ : one takes for $\epsilon$ the identity, and $\delta$ is given by the following chain of natural isomorphisms:

$$
\begin{gathered}
\left(F_{1}(A, I), H(X)\right) \simeq\left(F_{1}(A, I), F_{2}(I, X)\right) \simeq\left(F_{1}(A, I), I\right) \otimes\left(I, F_{2}(I, X)\right) \simeq \\
\simeq\left(F_{1}(A, I), F_{2}(A, I)\right) \otimes\left(F_{1}(I, X), F_{2}(I, X)\right)=F(A, I) \otimes F(I, X) \simeq \\
\simeq F((A, I) \otimes(I, X)) \simeq F(A, X)
\end{gathered}
$$

where the first isomorphism is provided by $\chi$, the third isomorphism is provided by $\chi$ on the first object and by $\varphi$ on the second object, and the other isomorphisms are canonical. We leave to the reader the (quite long) verification that $(\mathbf{R}, \mathbf{T}, \rho)$ is a 1-cell in $\mathbf{A c t}(\mathbf{C G})$ and $(\delta, \epsilon)$ is a 2 -cell in $\mathbf{P t}(\mathbf{C G})$.

Lemma 4.3. The 2-functor

$$
\rtimes: \operatorname{Act}(\mathbf{C G}) \rightarrow \operatorname{Pt}(\mathbf{C G})
$$

is biessentially surjective. 
Proof. Consider an object

$$
\mathbb{A} \underset{\mathrm{B}}{\stackrel{\mathrm{A}}{\rightleftarrows}} \mathbb{H}, \quad \alpha: \mathbf{A B} \Longrightarrow \mathbf{1}_{\mathbb{H}}
$$

in $\mathbf{P t}(\mathbf{C G})$. There is an action $\mathbb{H} \times \mathcal{N}(\mathbf{A}) \rightarrow \mathcal{N}(\mathbf{A})$ which sends a pair $(X,(A, a))$ on the object $\mathbf{B}(X) \otimes A \otimes \mathbf{B}(X)^{*}$ with structural map

$$
\begin{aligned}
\mathbf{A}\left(\mathbf{B}(X) \otimes A \otimes \mathbf{B}(X)^{*}\right) & \simeq \mathbf{A}(\mathbf{B}(X)) \otimes \mathbf{A}(A) \otimes \mathbf{A}\left(\mathbf{B}(X)^{*}\right) \stackrel{1 \otimes a \otimes 1}{\longrightarrow} \mathbf{A}(\mathbf{B}(X)) \\
\otimes \mathbf{A}\left(\mathbf{B}(X)^{*}\right) & \simeq I .
\end{aligned}
$$

We look for homomorphisms $\mathbf{F}$ and $\mathbf{H}$ as in the following diagram

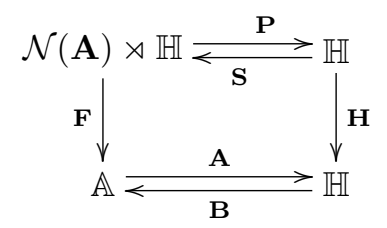

together with 2-cells $\varphi: \mathbf{F S} \Longrightarrow \mathbf{B H}$ and $\chi: \mathbf{A F} \Longrightarrow \mathbf{H P}$. We take as $\mathbf{H}$ the identity functor and we define $\mathbf{F}(A, a, X)=A \otimes \mathbf{B}(X)$. The monoidal structure of $\mathbf{F}$ comes from that of $\mathbf{B}$ in the following way:

$$
\begin{aligned}
\mathbf{F}((A, a, X) \otimes(B, b, Y)) & =A \otimes \mathbf{B}(X) \otimes B \otimes \mathbf{B}(X)^{*} \otimes \mathbf{B}(X \otimes Y) \\
& \simeq A \otimes \mathbf{B}(X) \otimes B \otimes \mathbf{B}(X)^{*} \otimes \mathbf{B}(X) \otimes \mathbf{B}(Y) \\
& \simeq A \otimes \mathbf{B}(X) \otimes B \otimes \mathbf{B}(Y) \\
& =\mathbf{F}(A, a, X) \otimes \mathbf{F}(B, B, Y)
\end{aligned}
$$

Finally, we put

$$
\begin{gathered}
\varphi_{X}: \mathbf{F}(\mathbf{S}(X))=I \otimes \mathbf{B}(X) \simeq \mathbf{B}(X) \\
\begin{aligned}
\chi_{(A, a, X)}: \mathbf{A}(\mathbf{F}(A, a, X)) & \simeq \mathbf{A}(A) \otimes \mathbf{A}(\mathbf{B}(X)) \stackrel{a \otimes \alpha_{X}}{\longrightarrow}>I \otimes X \simeq X \\
= & \mathbf{P}(A, a, X) .
\end{aligned}
\end{gathered}
$$

Since the kernel of $\mathbf{P}$ is equivalent to the kernel of $\mathbf{A}$, the short five lemma for categorical groups $[\mathbf{2 3}]$ implies that $\left(\mathbf{F}, 1_{\mathbb{H}}, \varphi, \chi\right)$ is an equivalence in $\mathbf{P t}(\mathbf{C G})$.

From the proof of the previous lemma, we can extract a 2-functor

$$
\mathcal{K}: \mathbf{P t}(\mathbf{C G}) \longrightarrow \operatorname{Act}(\mathbf{C G})
$$

quasi-inverse of the 2-functor $\rtimes$ : $\mathbf{A c t}(\mathbf{C G}) \rightarrow \mathbf{P t}(\mathbf{C G})$. The 2 -functor $\mathcal{K}$ acts as illustrated in the following diagram:

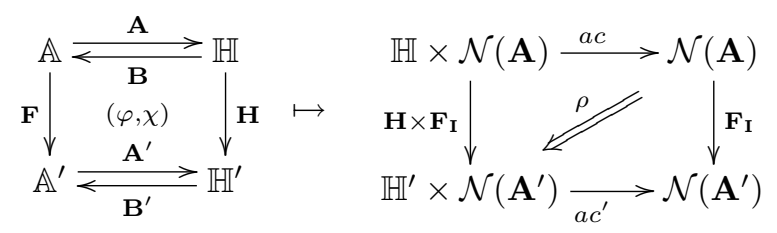


where $\mathbf{F}_{\mathbf{I}}$ is the restriction of $\mathbf{F}$ to the kernel, that is, $F_{I}(A, a)=\left(F(A), H(a) \cdot \chi_{A}\right)$, and the component of $\rho$ at $(X, A, a)$ is given by the composition

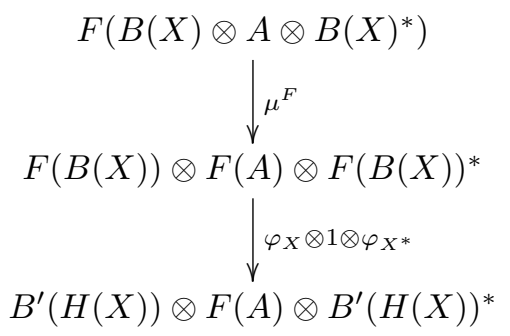

Finally, for a 2-cell $(\delta, \epsilon)$ in $\mathbf{P t}(\mathbf{C G}), \mathcal{K}(\delta, \epsilon)=(\epsilon, \bar{\delta})$, where $\bar{\delta}$ is the restriction of $\delta$ to $\mathbf{F}_{\mathbf{I}}$.

\subsection{Induced biequivalences}

There are forgetful 2-functors $\mathcal{U}_{*}: \mathbf{P t}(\mathbf{C G}) \rightarrow \mathbf{C G}$ and $\mathcal{O}: \mathbf{A c t}(\mathbf{C G}) \rightarrow \mathbf{C G}$ defined in the following diagrams
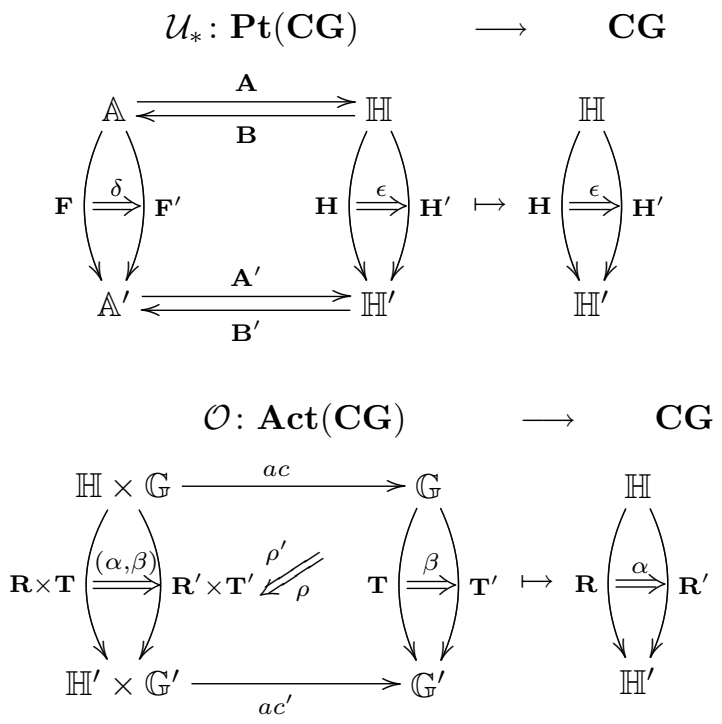

If $\mathbb{H}$ is a fixed categorical group, the strict 2-fibres $\mathbf{P t}(\mathbb{H})$ and $\operatorname{Act}(\mathbb{H})$ of $\mathcal{U}_{*}$ and $\mathcal{O}$ on $\mathbb{H}$ can be described in the following way:

- Objects, 1-cells and 2-cells in $\mathbf{P t}(\mathbb{H})$ are of the form

$$
\delta:(\mathbf{F}, \varphi, \chi) \Longrightarrow\left(\mathbf{F}^{\prime}, \varphi^{\prime}, \chi^{\prime}\right):(\mathbb{A}, \mathbf{A}, \mathbf{B}, \alpha) \rightrightarrows\left(\mathbb{A}^{\prime}, \mathbf{A}^{\prime}, \mathbf{B}^{\prime}, \alpha^{\prime}\right),
$$

where $(\mathbb{A}, \mathbb{H}, \mathbf{A}, \mathbf{B}, \alpha),\left(\mathbb{A}^{\prime}, \mathbb{H}, \mathbf{A}^{\prime}, \mathbf{B}^{\prime}, \alpha^{\prime}\right),\left(\mathbf{F}, 1_{\mathbb{H}}, \varphi, \chi\right),\left(\mathbf{F}^{\prime}, 1_{\mathbb{H}}, \varphi^{\prime}, \chi^{\prime}\right)$ and $\left(\delta, 1_{\mathbb{H}}\right)$ are objects, 1 -cells and 2-cells in $\mathbf{P t}(\mathbf{C G})$.

- Objects, 1-cells and 2-cells in $\mathbf{A c t}(\mathbb{H})$ are of the form

$$
\beta:(\mathbf{T}, \rho) \Longrightarrow\left(\mathbf{T}^{\prime}, \rho^{\prime}\right):(\mathbb{G}, a c) \rightrightarrows\left(\mathbb{G}^{\prime}, a c^{\prime}\right),
$$


Homology, Homotopy and Applications, vol. 8(1), 2006

where $(\mathbb{H}, \mathbb{G}, a c),\left(\mathbb{H}, \mathbb{G}^{\prime}, a c^{\prime}\right),\left(1_{\mathbb{H}}, \mathbf{T}, \rho\right),\left(1_{\mathbb{H}}, \mathbf{T}^{\prime}, \rho^{\prime}\right)$ and $\left(1_{\mathbb{H}}, \beta\right)$ are objects, 1cells and 2-cells in $\operatorname{Act}(\mathbf{C G})$.

Since the diagram

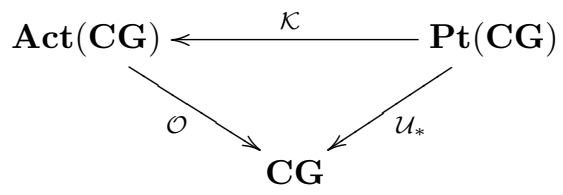

commutes, from Proposition 4.1 we get the following result.

Corollary 4.4. The biequivalence

$$
\mathcal{K}: \mathbf{P t}(\mathbf{C G}) \longrightarrow \operatorname{Act}(\mathbf{C G})
$$

restricts to a biequivalence

$$
\mathcal{K}^{\prime}: \operatorname{Pt}(\mathbb{H}) \longrightarrow \operatorname{Act}(\mathbb{H})
$$

Another forgetful 2-functor can be defined now:

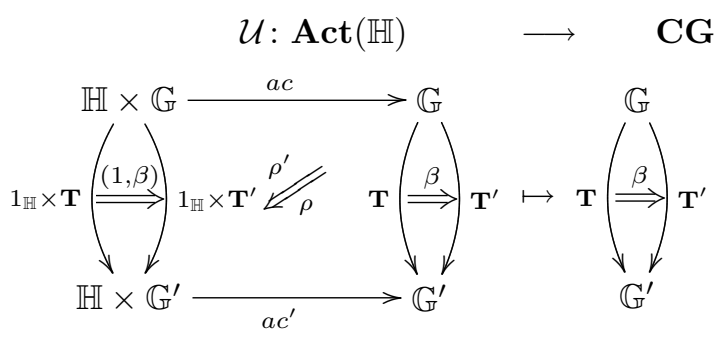

Composing $\mathcal{K}^{\prime}$ and $\mathcal{U}$, we get a 2 -functor

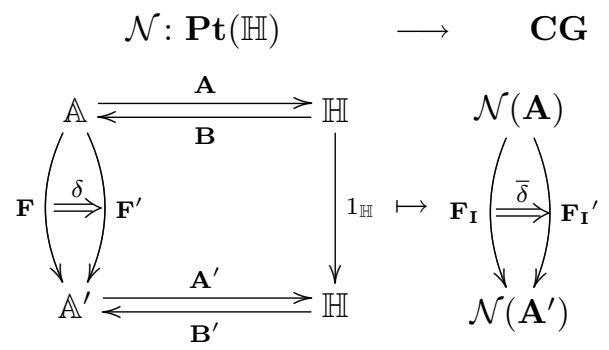

where $\bar{\delta}: \mathbf{F}_{\mathbf{I}} \Longrightarrow \mathbf{F}_{\mathbf{I}}^{\prime}$ are the restrictions of $\delta: \mathbf{F} \Longrightarrow \mathbf{F}^{\prime}$ to the kernel. Now, let us fix a categorical group $\mathbb{G}$ and look at the 2-fibres of $\mathcal{U}$ and $\mathcal{N}$ on $\mathbb{G}$ :

- The strict 2-fibre of $\mathcal{U}$ on $\mathbb{G}$ is the groupoid (= locally discrete 2-groupoid) whose objects are actions $a c: \mathbb{H} \times \mathbb{G} \rightarrow \mathbb{G}$ of $\mathbb{H}$ on $\mathbb{G}$ and whose arrows are natural transformations $\rho: a c \Longrightarrow a c^{\prime}$ such that $\left(1_{\mathbb{H}}, 1_{\mathbb{G}}, \rho\right)$ is a 1-cell in $\operatorname{Act}(\mathbf{C G})$.

- The homotopy 2-fibre of $\mathcal{N}$ on $\mathbb{G}$ is precisely the 2-groupoid of split extensions $\mathbf{E x t}_{\text {split }}(\mathbb{H}, \mathbb{G})$ described in Section 3.2. 
Corollary 4.5. The biequivalence

$$
\mathcal{K}^{\prime}: \mathbf{P t}(\mathbb{H}) \longrightarrow \operatorname{Act}(\mathbb{H})
$$

restricts to a biequivalence

$$
\mathcal{K}^{\prime \prime}:=\operatorname{Ext}_{\text {split }}(\mathbb{H}, \mathbb{G}) \longrightarrow \operatorname{Act}(\mathbb{H}, \mathbb{G}) .
$$

Proof. Since $\mathcal{N}=\mathcal{U} \cdot \mathcal{K}^{\prime}$, the biequivalence $\mathcal{K}^{\prime}$ restricts to a biequivalence between the strict 2 -fibres on $\mathbb{G}$. Moreover, the 2 -functor $\mathcal{N}$ is a fibration of 2-groupoids, so that the strict fibre and the homotopy fibre are biequivalent [13].

\section{Universal properties of the holomorph of a categorical group}

\subsection{Birepresentability}

To generalize the first universal property of the holomorph to our context, we need the following birepresentability criterion, established in [25].

Proposition 5.1. Let $\mathcal{C}$ be a bicategory. Consider the 2-category Cat of small categories and a morphism

$$
\mathcal{F}: \mathcal{C}^{o p} \rightarrow \text { Cat, }
$$

i.e. $\mathcal{F}$ preserves composition and identities up to natural coherent invertible 2-cells (superscript "op" refers to notation in [25], meaning that $\mathcal{F}$ inverts 1-cells but not 2-cells). The following conditions are equivalent:

1. there exist an object $R$ of $\mathcal{C}$ and a natural equivalence $\theta: \mathcal{F} \Longrightarrow \mathcal{C}(-, R)$, i.e. an equivalence $\theta$ in $\operatorname{Hom}\left(\mathcal{C}^{o p}\right.$, Cat $)$

2. there exist an object $R$ of $\mathcal{C}$ and an object $a \in \mathcal{F}(R)$ such that

- for every object $H$ of $\mathcal{C}$, and for every object $x \in \mathcal{F}(H)$, there exist an arrow $h: H \rightarrow R$ in $\mathcal{C}$ and an isomorphism $u: x \rightarrow \mathcal{F}(h)(a)$ in $\mathcal{F}(H)$

- given two arrows $h^{\prime}: H \rightarrow R, \alpha: \mathcal{F}(h)(a) \rightarrow \mathcal{F}\left(h^{\prime}\right)(a)$, there exists a unique 2-cell $\sigma: h \Longrightarrow h^{\prime}$ such that the component at a of the natural transformation $\mathcal{F}(\sigma)$ is $\alpha$.

The aim of this section is to show that split extensions of categorical groups are represented by the holomorph split extension. In order to present the result, a few preliminary notions are needed.

\subsection{Pullbacks of categorical groups}

Given two homomorphisms of categorical groups $\mathbf{F}: \mathbb{B} \rightarrow \mathbb{A}$ and $\mathbf{G}: \mathbb{C} \rightarrow \mathbb{A}$, what we call here their pullback is a universal (in the sense of bilimits) 4 -tuple $\left(\mathbb{P}, \mathbf{F}^{\prime}, \mathbf{G}^{\prime}, \varpi\right)$ 
represented by the following diagram in $\mathbf{C G}$

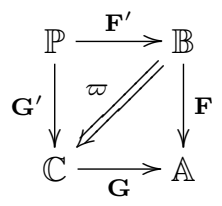

This means that for any other diagram

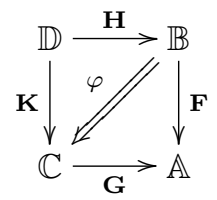

there is a homomorphism $\mathbf{D}: \mathbb{D} \rightarrow \mathbb{P}$, and two 2-cells $\varphi_{H}: \mathbf{H} \Longrightarrow \mathbf{F}^{\prime} \mathbf{D}$ and $\varphi_{K}: \mathbf{K} \Longrightarrow \mathbf{G}^{\prime} \mathbf{D}$ such that the following diagram commutes

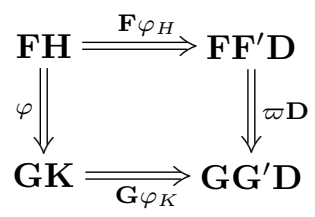

Moreover, for any other triple $\left(\mathbf{D}^{\prime}, \varphi_{H}^{\prime}, \varphi_{K}^{\prime}\right)$ satisfying the same property, there is a unique 2-cell $\psi: \mathbf{D} \Longrightarrow \mathbf{D}^{\prime}$ such that the following diagrams commute
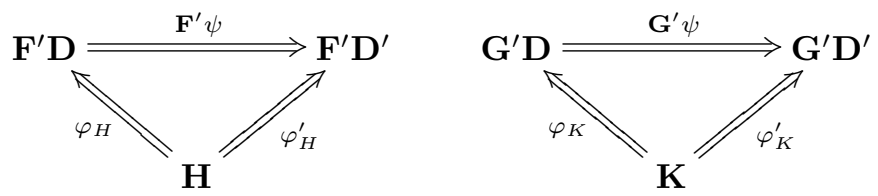

In [6], an explicit description of the pullback is given: $\mathbb{P}$ is the comma-groupoid whose objects are triples $(B \in \mathbb{B}, u: F B \rightarrow G C, C \in \mathbb{C})$; an arrow in $\mathbb{P}$ is a pair $f_{B}: B \rightarrow B^{\prime}, f_{C}: C \rightarrow C^{\prime}$ such that the following square commutes

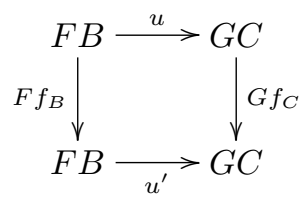

Tensor products of $\mathbb{B}$ and $\mathbb{C}$ extend naturally to $\mathbb{P}$, so that $\mathbb{P}$ is indeed a categorical group. Homomorphism $\mathbf{F}^{\prime}$ forgets the $\mathbb{C}$ side and $\mathbf{G}^{\prime}$ forgets the $\mathbb{B}$ side, and the 2-cell $\varpi$ is given by $\varpi_{(B, u, C)}=u$.

Many properties of pullbacks can be revisited in dimension 2 just using the universal property of the bilimit. The relevant one for our purpose is that, given a homomorphism $\mathbf{F}: \mathbb{K} \rightarrow \mathbb{H}$ of categorical groups and a point

$$
\mathbb{A} \underset{\mathbf{B}}{\stackrel{\mathbf{A}}{\rightleftarrows}} \mathbb{H}, \quad \alpha: \mathbf{A B} \Longrightarrow \mathbf{1}_{\mathbb{H}}
$$


over $\mathbb{H}$, pulling back along $\mathbf{F}$ produces a point over $\mathbb{K}$. Once an explicit description of the pullback is chosen, this produces a 2 -functor

$$
\mathbf{F}^{*}: \mathbf{P t}(\mathbb{H}) \rightarrow \mathbf{P t}(\mathbb{K}) .
$$

Moreover, such a 2-functor induces a 2-functor

$$
\operatorname{Ext}_{\text {split }}(\mathbf{F}, \mathbb{G}): \operatorname{Ext}_{\text {split }}(\mathbb{H}, \mathbb{G}) \rightarrow \operatorname{Ext}_{\text {split }}(\mathbb{K}, \mathbb{G}) .
$$

\subsection{The holomorph of a categorical group}

Let $\mathbb{G}$ be a categorical group. The holomorph of $\mathbb{G}$, denoted $\mathcal{H o l}(\mathbb{G})$, is the semidirect product $\mathbb{G} \rtimes \mathcal{E} q(\mathbb{G})$, with action given by evaluation. In other words, the holomorph of $\mathbb{G}$ fits into a split extension of categorical groups

$$
\mathcal{H}=(\mathbb{G} \longrightarrow \mathcal{H o l}(\mathbb{G}) \rightleftarrows \mathcal{E} q(\mathbb{G})),
$$

that is, an object of $\operatorname{Ext}_{\text {split }}(\mathcal{E} q(\mathbb{G}), \mathbb{G})$.

Recall from $[\mathbf{2}]$ that the classifying category of a bicategory $\mathcal{C}$ has the same objects of $\mathcal{C}$, and arrows are 2-isomorphism classes of 1-cells of $\mathcal{C}$. Let us denote by $\mathbf{E X T}_{\text {split }}(\mathbb{H}, \mathbb{G})$ the classifying category of $\mathbf{E x t}_{\text {split }}\left(\mathbb{H}, \mathbb{G}\right.$ ) (in fact, $\mathbf{E X T}_{\text {split }}(\mathbb{H}, \mathbb{G}$ ) is a groupoid, since it is the classifying category of a 2-groupoid). The biequivalence

$$
\mathcal{K}^{\prime \prime}: \operatorname{Ext}_{\text {split }}(\mathbb{H}, \mathbb{G}) \longrightarrow \operatorname{Act}(\mathbb{H}, \mathbb{G})
$$

of Corollary 4.5 induces an equivalence of groupoids

$$
\operatorname{EXT}_{\text {split }}(\mathbb{H}, \mathbb{G}) \longrightarrow \operatorname{Act}(\mathbb{H}, \mathbb{G}) .
$$

Moreover, the previous discussion on pullbacks of categorical groups allows us to convert this equivalence into a natural equivalence

$$
\operatorname{EXT}_{\text {split }}(-, \mathbb{G}) \Longrightarrow \operatorname{Act}(-, \mathbb{G}) .
$$

Finally, let us observe that the correspondence between actions of $\mathbb{H}$ on $\mathbb{G}$ and homomorphisms $\mathbb{H} \rightarrow \mathcal{E} q(\mathbb{G})$, already quoted in Section 3.3, is indeed part of an equivalence

$$
\operatorname{Act}(\mathbb{H}, \mathbb{G}) \rightarrow \operatorname{Hom}_{\mathrm{CG}}(\mathbb{H}, \mathcal{E} q(\mathbb{G})) .
$$

Once again, this equivalence can be converted into a natural equivalence

$$
\operatorname{Act}(-, \mathbb{G}) \rightarrow \operatorname{Hom}_{\mathbf{C G}}(-, \mathcal{E} q(\mathbb{G})) .
$$

Putting together the previous natural equivalences and applying the birepresentability criterion of Section 5.1 to the 2-functor

$$
\mathbf{E X T}_{\text {split }}(-, \mathbb{G}): \mathbf{C G}^{o p} \rightarrow \mathbf{C a t},
$$

we get the following universal property of the holomorph.

Proposition 5.2. Consider two categorical groups $\mathbb{G}$ and $\mathbb{H}$. 
1. For any split extension

$$
\mathcal{E}=\left(\mathbb{G} \longrightarrow \mathbb{A} \underset{\mathrm{B}}{\stackrel{\mathrm{A}}{\rightleftarrows}} \mathbb{H}, \alpha: \mathbf{A B} \Longrightarrow \mathbf{1}_{\mathbb{H}}\right),
$$

there is a 1-cell $\mathbf{\Sigma}: \mathbb{H} \rightarrow \mathcal{E} q(\mathbb{G})$ in $\mathbf{C G}$ and an isomorphism between the given split extension and the split extension obtained pulling back the holomorph split extension

$$
\mathcal{H}=(\mathbb{G} \longrightarrow \mathcal{H o l}(\mathbb{G}) \longleftrightarrow \mathcal{E} q(\mathbb{G}))
$$

along $\boldsymbol{\Sigma}$, say $u: \mathcal{E} \rightarrow \mathbf{E X T}_{\text {split }}(\boldsymbol{\Sigma}, \mathbb{G})(\mathcal{H})$.

2. Moreover, for any other 1-cell $\boldsymbol{\Sigma}^{\prime}: \mathbb{H} \rightarrow \mathcal{E} q(\mathbb{G})$ in $\mathbf{C G}$ and for any other isomorphism $u^{\prime}: \mathcal{E} \rightarrow \mathbf{E X T}_{\text {split }}\left(\boldsymbol{\Sigma}^{\prime}, \mathbb{G}\right)(\mathcal{H})$, there is a unique 2-cell $\sigma: \boldsymbol{\Sigma} \Longrightarrow \boldsymbol{\Sigma}^{\prime}$ in CG such that $\mathbf{E X T}_{\text {split }}(\sigma, \mathbb{G})(\mathcal{H}) \cdot u=u^{\prime}$.

Remark 5.3. In spite of the fact that CG is a 2-category, in order to apply the 2dimensional representability criterion of Section 5.1 , it is necessary to pass through a quotient that identifies isomorphic 1-cells. Indeed, representability of $\mathbf{E x t}_{\text {split }}(-, \mathbb{G})$ lives in a 3-categorical context when categorical groups are seen as bicategories with only one object. Nevertheless, we decided to avoid 3-categorical complications because the 2-dimensional representability recaptures the universality of the holomorph construction we are interested in. The reason is that the wider flexibility available in 3-categories trivializes because of local discreteness of categorical group actions.

\subsection{Holomorph and inner autoequivalences}

Composing the biequivalence $\rtimes: \mathbf{A c t}(\mathbf{C G}) \rightarrow \mathbf{P t}(\mathbf{C G})$ of Section 4.2 with the forgetful 2-functor $\mathcal{U}^{*}: \mathbf{P t}(\mathbf{C G}) \rightarrow \mathbf{C G}, \mathcal{U}^{*}(\mathbb{A}, \mathbb{H}, \mathbf{A}, \mathbf{B})=\mathbb{A}$, we get a 2 -functor

$$
\rtimes: \operatorname{Act}(\mathbf{C G}) \longrightarrow \mathbf{C G} .
$$

Moreover, the conjugation of a categorical group described in Section 3.3 plainly extends to a 2-functor

$$
\mathcal{C}: \mathbf{C G} \longrightarrow \operatorname{Act}(\mathbf{C G}) \text {. }
$$

Proposition 5.4. The 2-functor $\mathcal{C}: \mathbf{C G} \rightarrow \mathbf{A c t}(\mathbf{C G})$ is a right biadjoint to the 2-functor $\rtimes: \mathbf{A c t}(\mathbf{C G}) \rightarrow \mathbf{C G}$.

Proof. We refer to [25] for the definition of biadjunction and we limit ourselves to the description of the natural equivalence between hom-categories

$$
\operatorname{Hom}_{\mathbf{A c t}(\mathbf{C G})}(a c: \mathbb{H} \times \mathbb{G} \rightarrow \mathbb{G}, \mathcal{C}(\mathbb{A})) \simeq \operatorname{Hom}_{\mathbf{C G}}(\mathbb{G} \rtimes \mathbb{H}, \mathbb{A}) .
$$

Given a morphism of actions

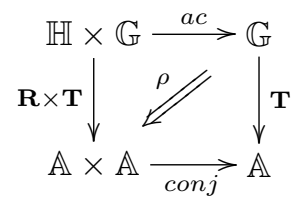


we define $\boldsymbol{\Gamma}: \mathbb{G} \rtimes \mathbb{H} \rightarrow \mathbb{A}$ by $\boldsymbol{\Gamma}(A, X)=\mathbf{T}(A) \otimes \mathbf{R}(X)$. The monoidal structure of $\boldsymbol{\Gamma}$ is given by

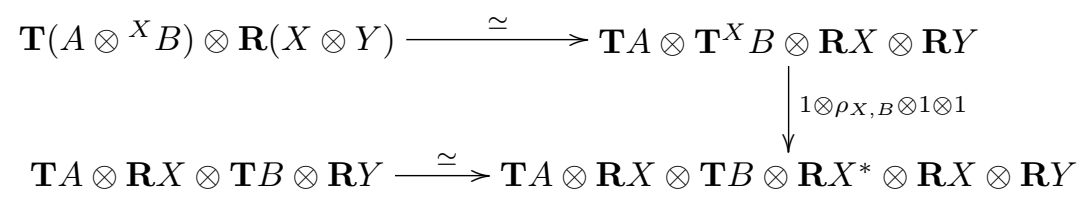

Given a 2-cell $\left(\alpha: \mathbf{R} \Rightarrow \mathbf{R}^{\prime}, \beta: \mathbf{T} \Rightarrow \mathbf{T}^{\prime}\right)$ in $\mathbf{A c t}(\mathbf{C G})$, we get a 2-cell $\boldsymbol{\Gamma} \Rightarrow \boldsymbol{\Gamma}^{\prime}$ in $\mathbf{C G}$ by $\beta_{A} \otimes \alpha_{X}: \mathbf{T} A \otimes \mathbf{R} X \rightarrow \mathbf{T}^{\prime} A \otimes \mathbf{R}^{\prime} X$. Conversely, given a morphism $\boldsymbol{\Gamma}: \mathbb{G} \rtimes \mathbb{H} \rightarrow$ $\mathbb{A}$, we get $\mathbf{R}: \mathbb{H} \rightarrow \mathbb{A}$ and $\mathbf{T}: \mathbb{G} \rightarrow \mathbb{A}$ precomposing $\boldsymbol{\Gamma}$ with the canonical injections of $\mathbb{H}$ and $\mathbb{G}$ into the semidirect product. Finally, consider $(X, B) \in \mathbb{H} \times \mathbb{G}$. We obtain the natural transformation $\rho$ tensoring the following composition with $\mathbf{R} X^{*}$, which is constructed using the monoidal structure of $\boldsymbol{\Gamma}$

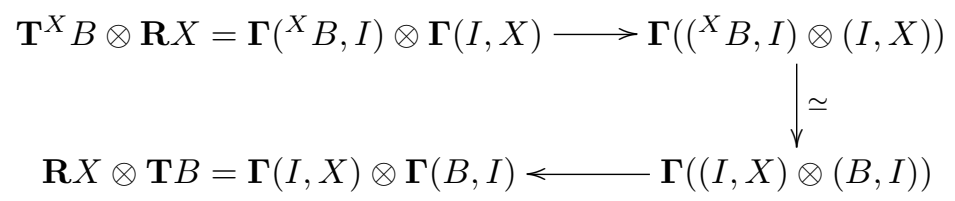

Now, observe that the canonical morphisms

$$
\mathbb{G} \stackrel{i n_{1}}{\longrightarrow} \mathcal{H o l}(\mathbb{G})<\stackrel{i n_{2}}{\leftarrow} \mathcal{E} q(\mathbb{G})
$$

are equipped with a natural family of 2-cells

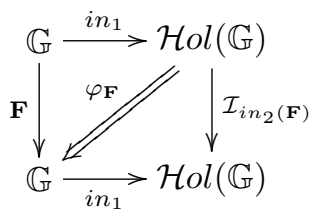

where $\mathbf{F}$ varies in $\mathcal{E} q(\mathbb{G})$. If, in the equivalence described in the proof of Proposition 5.4 , we take as the action the evaluation $\mathcal{E} q(\mathbb{G}) \times \mathbb{G} \rightarrow \mathbb{G}$, we obtain the second universal property of the holomorph: for any triple

$$
\mathbb{G} \stackrel{\mathbf{T}}{\longrightarrow} \mathbb{A} \longleftrightarrow \mathbf{R} \mathcal{E} q(\mathbb{G})
$$

in CG equipped with a natural family of 2-cells

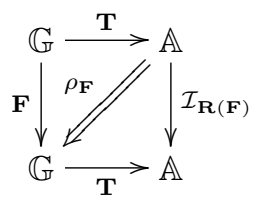


there is a triple $(\alpha, \boldsymbol{\Gamma}, \beta)$ in $\mathbf{C G}$

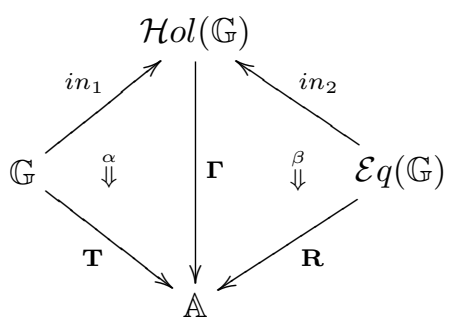

which renders commutative the following diagram of 2-cells, where the unlabelled 2-cell is determined by the monoidal structure of $\boldsymbol{\Gamma}$

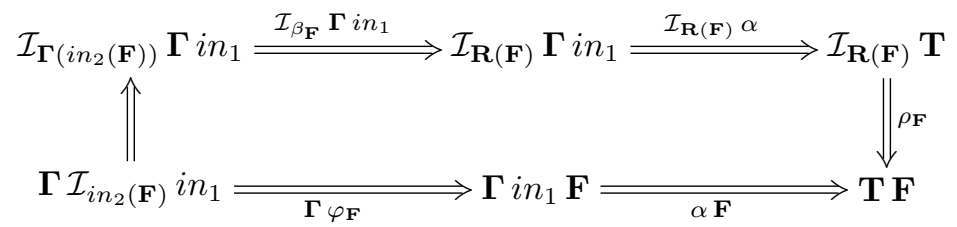

Moreover, if $\left(\alpha^{\prime}, \boldsymbol{\Gamma}^{\prime}, \beta^{\prime}\right)$ is another such a triple, then there is a unique 2-cell $\omega: \boldsymbol{\Gamma} \Rightarrow$ $\boldsymbol{\Gamma}^{\prime}$ in $\mathbf{C G}$ making commutative the following diagrams
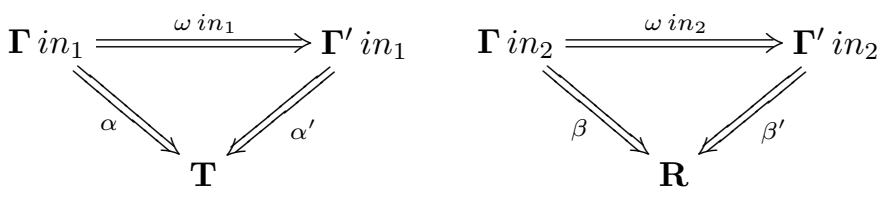

\section{Acknowledgements}

We would like to thank G. Janelidze for some helpful discussions on split extensions of groups. Financial support by INDAM and FNRS grant 1.5.121.6 are gratefully acknowledged.

\section{References}

[1] J. Baez, and A. Lauda, Higher-dimensional algebra. V: 2-Groups, Theory and Applications of Categories 12 (2004), pp. 423-491, http://www.tac.mta.ca/tac/.

[2] J. Bénabou, Introduction to bicategories, Springer Lecture Notes in Mathematics 40 (1967), pp. 1-77.

[3] F. Borceux, G. Janelidze, and G. M. Kelly, Internal object actions, Comment. Math. Univ. Carolinae 46 (2005), pp. 235-155.

[4] F. Borceux, G. Janelidze, and G. M. Kelly, On the representability of actions in a semi-abelian category, Theory and Applications of Categories 14 (2005), pp. 244-286, http://www.tac.mta.ca/tac/. 
[5] D. Bourn and G. Janelidze, Protomodularity, descent, and semidirect product, Theory and Applications of Categories 4 (1998), pp. 37-46, http://www.tac.mta.ca/tac/.

[6] D. Bourn and E. M. Vitale, Extensions of symmetric cat-groups, Homology, Homotopy and Applications 4 (2002), pp. 103-162, http://intlpress.com/HHA/v4/n1/a8/.

[7] L. Breen, Théorie de Schreier supérieure, Ann. scient. Éc. Norm. Sup. $4^{e}$ série, 25 (1992), pp. 465-514.

[8] P. Carrasco and A. Cegarra, Schreier theory for central extensions of categorical groups, Commmunications in Algebra 24 (1996), pp. 4059-4112.

[9] P. Carrasco and A. R. Garzón, Obstruction theory for extensions of categorical groups, Applied Categorical Structures 12 (2004), pp. 35-61.

[10] P. Carrasco and J. Martínez Moreno, Categorical G-crossed modules and 2-fold extensions, Journal of Pure and Applied Algebra 163 (2001), pp. 235-257.

[11] P. Carrasco, A. R. Garzón, and E.M. Vitale, On categorical crossed modules, Preprint (2005).

[12] A. del Río, J. Martínez Moreno, and E. M. Vitale, Chain complexes of symmetric categorical groups, Journal of Pure and Applied Algebra 196 (2005), pp. 279-312.

[13] J. W. Duskin, R. W. Kieboom, and E. M. Vitale, Morphisms of 2-groupoids and low-dimensional cohomology of crossed modules, Fields Institute Communications 43 (2004), pp. 227-241.

[14] A. Fröhlich and C. T. C. Wall, Graded monoidal categories, Compositio Mathematica 28 (1974), pp. 229-285.

[15] A. R. Garzón and H. Inassaridze, Semidirect products of categorical groups. Obstruction theory, Homology, Homotopy and Applications 3 (2001), pp. 111138, http://intlpress. com/HHA/v3/n1/a6/.

[16] A. R. Garzón, H. Inassaridze, and A. del Río, Derivations of categorical groups, Theory and Applications of Categories 13 (2004), pp. 86-105, http://www.tac.mta.ca/tac/.

[17] A. R. Garzón and A. del Río, The Whitehead categorical group of derivations, Georgian Mathematics Journal 9 (2002), pp. 709-721.

[18] A. R. Garzón and A. del Río, Low-dimensional cohomology for categorical groups, Cahiers de Top. et Géom. Diff. Catégoriques 44 (2003), pp. 247-280.

[19] G. Janelidze, Internal crossed modules, Georgian Mathematics Journal 10 (2003), pp. 99-114.

[20] A. Joyal and R. Street, Braided tensor categories, Advances in Mathematics 82 (1991), pp. 20-78.

[21] S. Kasangian and E. M. Vitale, Factorization systems for symmetric cat-groups, Theory and Applications of Categories 7 (2000), pp. 47-70, http://www.tac.mta.ca/tac/. 
[22] A. G. Kurosh, The theory of groups, vol. 1, Chelsea Publishing Company, New York, 1955.

[23] A. Rousseau, Bicatégories monoidales et extensions de Gr-catégories, Homology, Homotopy and Applications 5 (2003), pp. 437-547, http://intlpress . com/HHA/v5/n1/a19/.

[24] H. X. Sinh, Gr-catégories, Université Paris 7, Thèse de doctorat, 1975.

[25] R. Street, Fibrations in bicategories, Cahiers de Top. et Géom. Diff. Catégoriques 21 (1980), pp. 111-160.

[26] E. M. Vitale, A Picard-Brauer exact sequence of categorical groups, Journal of Pure and Applied Algebra 175 (2002), p. 387-408.

Stefano Kasangian Stefano.Kasangian@mat.unimi.it

Giuseppe Metere metere@mat.unimi.it

Dipartimento di Matematica

Università di Milano

Via Saldini 50

I 20133 Milano, Italy

Enrico M. Vitale vitale@math.ucl.ac.be

Département de Mathématique Pure et Appliquée

Université catholique de Louvain

Chemin du Cyclotron 2

B 1348 Louvain-la-Neuve, Belgium

This article is available at http://intlpress.com/HHA/v8/n1/a4/ 